\title{
Nonrelativistic perpendicular shocks of young supernova remnants - shock structure and particle injection processes
}

\author{
Jacek Niemiec*, Artem Bohdan, Oleh Kobzar, \\ Instytut Fizyki Jqdrowej PAN, ul. Radzikowskiego 152, 31-342 Kraków, Poland \\ E-mail: jacek.niemiec@ifj.edu.pl

\section{Martin Pohl} \\ Institute of Physics and Astronomy, University of Potsdam, Karl-Liebknecht-Strasse 24/25, \\ 14476 Potsdam, Germany \\ DESY, Platanenallee 6, 15738 Zeuthen, Germany
}

\begin{abstract}
The shock formation, their electromagnetic structure, and the injection of suprathermal particles into the first-order Fermi acceleration processes at collisionless shocks constitute important problems of high-energy astrophysics. We report on recent studies of high Mach-number nonrelativistic perpendicular shocks with fully kinetic two-dimensional particle-in-cell simulations in applications to forward shocks of young supernova remnants. We discuss a nonlinear shock structure mediated by electromagnetic Weibel-type filamentation instabilities and demonstrate the cyclic shock reformation and shock rippling processes known before only for low Mach-number shocks. We also analyse conditions leading to particle heating and pre-acceleration. We confirm electron injection through shock-surfing acceleration in the foot of high Mach-number shocks. We show that the injection efficiency is not stationary and varies with the phase of the shock reformation. It also nonlinearly depends on the upstream plasma temperature. Furthermore, we demonstrate that further electron energization processes in the shock ramp strongly depend on the configuration of the uniform perpendicular magnetic field with respect to the simulation plane, highlighting a need for a fully three-dimensional kinetic studies.
\end{abstract}

35th International Cosmic Ray Conference - ICRC2017

10-20 July, 2017

Bexco, Busan, Korea

${ }^{*}$ Speaker. 


\section{Introduction}

The first-order Fermi acceleration (aka diffusive shock acceleration, DSA) process operating at collisionless nonrelativistic shocks of supernova remnants (SNRs) is widely believed to be the source of Galactic cosmic rays (CRs) with energies up to the knee at $\sim 10^{15} \mathrm{eV}$. The most tantalizing unresolved question in DSA theory is the particle injection problem: since the width of the shock transition layer is of the order of several proton gyroradii, some pre-acceleration is required for thermal particles. The problem is more severe for electrons on account of their lower mass and consequently smaller Larmor radii and inertial lengths, compared to protons.

Here we study perpendicular shocks in a regime of high Alfvénic and sonic Mach numbers, $M_{\mathrm{A}} \gtrsim 30$ and $M_{\mathrm{s}} \gtrsim 50$, as appropriate for forward shocks of young SNRs. The physics of such shocks is governed by ion reflection at the shock ramp that leads to Weibel-type filamentation instability generating magnetic filaments and mediating the shock transition. As shown in our recent study [1], the shocks are non-stationary and cyclically self-reform on the time scale of the ion gyration in the upstream magnetic field. As in low-Mach-number shocks, the reformation is driven by a non-steady ion reflection. At the same time, spatial modulations of the reflected ion fraction along the shock surface may cause the shock surface rippling, as in the scenario known for shocks with low Mach numbers [2, 3].

It is known that the ion injection at perpendicular shocks can result from shock-surfing acceleration (SSA), in which shock-reflected particles performing half a gyro cycle upstream are energized through motion along the convective electric field. Similar injection mechanism can work for electrons at high-Mach-number shocks due to their interaction with strong electrostatic waves resulting from the electrostatic two-stream instability, also known as Buneman instability [4]. This instability is excited in the shock foot in interaction between cold incoming electrons and reflected ions. Electrons that are captured in electrostatic potential wells of nonlinear waves, can be accelerated in perpendicular direction by the convective electric field. Their multiple rapid interactions in the Buneman wave region give rise to a so-called electron SSA and produce supra-thermal tails in the electron spectra. Particle-in-cell (PIC) computer simulations in 1D and 2D confirm this picture $[5,6,7,8,1]$. However, our understanding of the efficiency of SSA and its dependence on the ion-to-electron mass ratio, the plasma temperature, the magnetic-field orientation with respect to the simulation plane, etc., is still incomplete.

Our earlier study of perpendicular high-Mach-number shocks revealed efficient electron heating that arises from Buneman modes in the shock foot, but very little acceleration that leads to the creation of supra-thermal spectral tail. Here we report results of our recent work [9] that uses 2D PIC simulations to analyse conditions allowing for efficient electron pre-acceleration. We study shocks whose Alfvénic Mach number, $M_{\mathrm{A}}$, satisfies both a threshold condition for the excitation of electrostatic waves in the shock foot and the condition enabling electron trapping in the strong electrostatic wave potential [7]. We consider low plasma betas, $\beta_{\mathrm{p}} \leq 0.5$, to complement previous $2 \mathrm{D}$ shock investigations $[7,8,10,1]$. For the fixed ion-to-electron mass ratio, $m_{i} / m_{e}=100$, used in our simulations, we investigate the impact of the magnetic-field configuration, namely, the angle between the regular magnetic field and the simulation plane on the electron energization. We expect that these simulations may give us better understanding of acceleration processes in high-Mach-number shocks under fully three-dimensional geometry. 


\begin{tabular}{ccccccc}
\hline \hline Run & $\varphi$ & $\beta_{p}$ & $L_{y}\left(\lambda_{s i}\right)$ & $M_{A}$ & $M_{s}$ & NTEF [\%] \\
\hline \hline A1 & $0^{\circ}$ & 0.0005 & 24 & 31.7 & 1550 & $0.2 \pm 0.1$ \\
A2 & $0^{\circ}$ & 0.5 & 24 & 31.7 & 49 & $0.7 \pm 0.1$ \\
\hline B1 & $45^{\circ}$ & 0.0005 & 24 & 31.7 & 1550 & $0.2 \pm 0.1$ \\
B2 & $45^{\circ}$ & 0.5 & 24 & 31.7 & 49 & $0.5 \pm 0.1$ \\
\hline C1 & $90^{\circ}$ & 0.0005 & 12 & 35.5 & 1581 & $4 \pm 1$ \\
C2 & $90^{\circ}$ & 0.5 & 12 & 35.5 & 55 & $7 \pm 1$
\end{tabular}

Table 1: Parameters of the simulations and derived shock and downstream properties. $L_{y}-$ transverse size of the simulation box in units of the ion skin depth, $\lambda_{\mathrm{si}} ; M_{A}, M_{s}$ - Alfvénic and sonic Mach numbers of the shock in the upstream frame, respectively, NTEF - nonthermal electron fraction. All runs use the electron skin depth of $\lambda_{\mathrm{se}}=20 \Delta$, where $\Delta$ is the cell size, 20 particles per cell per particle species for both plasma slabs, the ion-electron mass ratio $m_{i} / m_{e}=100$, and the plasma magnetization $\omega_{\mathrm{pe}} / \Omega_{\mathrm{e}}=12$. The simulation time, $t=8.125 \Omega_{i}^{-1}$, is chosen to cover at least 3 shock reformation cycles.

\section{Simulation setup}

The simulation setup is presented in Figure 1. A collision of two counter-streaming electronion plasma beams leads to the formation of two shocks propagating in opposite directions. The plasma is contained in the $x-y$ plane with open $x$-boundaries and periodic boundary conditions in the $y$-direction. The plasma beams are continuously injected from both sides of the simulation box and carry a uniform magnetic field, $\mathbf{B}_{\mathbf{0}}$, which is perpendicular to the plasma flow. The field thus lies in the $y-z$ plane but forms an angle $\varphi$ with the $y$-axis. A motional electric field $\mathbf{E}=-\mathbf{v} \times \mathbf{B}_{\mathbf{0}}$ is also initialized in the left and right stream, with $\mathbf{v}=\mathbf{v}_{\mathrm{L}}$ or $\mathbf{v}=\mathbf{v}_{\mathrm{R}}$, respectively. The counterstreaming plasma beams have equal densities and move with equal absolute velocities, $v_{\mathrm{L}}=0.2 c=$ $v_{\mathrm{R}}$. A relative collision velocity is then $v_{\text {rel }} \simeq 0.38 c$, where $c$ is the speed of light. Restricted by available computational resources, we use a $2 \mathrm{D} 3 \mathrm{~V}$ model, i.e., we follow particle trajectories in the $x y$ plane but keep track of all three components of particle velocities and electromagnetic fields. Since, as we show, in such a geometry the physics depends on the orientation of the initially

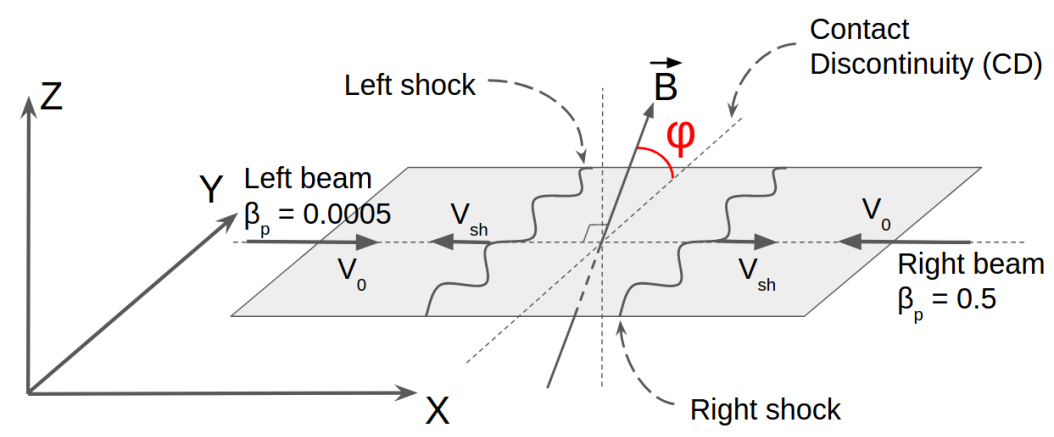

Figure 1: Illustration of the simulation setup. 
uniform perpendicular magnetic field with respect to the simulation plane, we carry out simulations for three values of the angle $\varphi$, namely in-plane magnetic field $\varphi=0^{\circ}$ (runs A1 and A2), $\varphi=45^{\circ}$ (runs B1 and B2), and out-of-plane magnetic field $\varphi=90^{\circ}$ (runs $\mathrm{C} 1$ and $\mathrm{C} 2$ ). For a given angle $\varphi$, the temperatures of the two beams are different. We set the plasma beta (the ratio of the plasma pressure to the magnetic pressure) in the left slab to $\beta_{\mathrm{p}, \mathrm{L}}=0.0005$ and in the right slab to $\beta_{\mathrm{p}, \mathrm{R}}=0.5$. Thus the difference in the sonic Mach numbers, $M_{\mathrm{S}}$, of the two shocks is $\sim 30$. Run-specific parameters are listed in Table 1. Note, that the sonic and Alfvénic Mach numbers of the shocks in our simulations satisfy both the unstable $\left(M_{\mathrm{s}} \geq 6\right)$ and the trapping condition $\left(M_{\mathrm{A}} \gtrsim 25.8\right)$. Hence, in all cases we should expect efficient electron acceleration.

The code used in this study is a 2D3V-adapted and modified version of the relativistic electromagnetic particle code TRISTAN with MPI-based parallelization [11, 12]. The numerical model is essentially the same as that used in [1]. A notable addition is the possibility to follow individual selected particle trajectories, which allows us to study particle acceleration processes in detail.

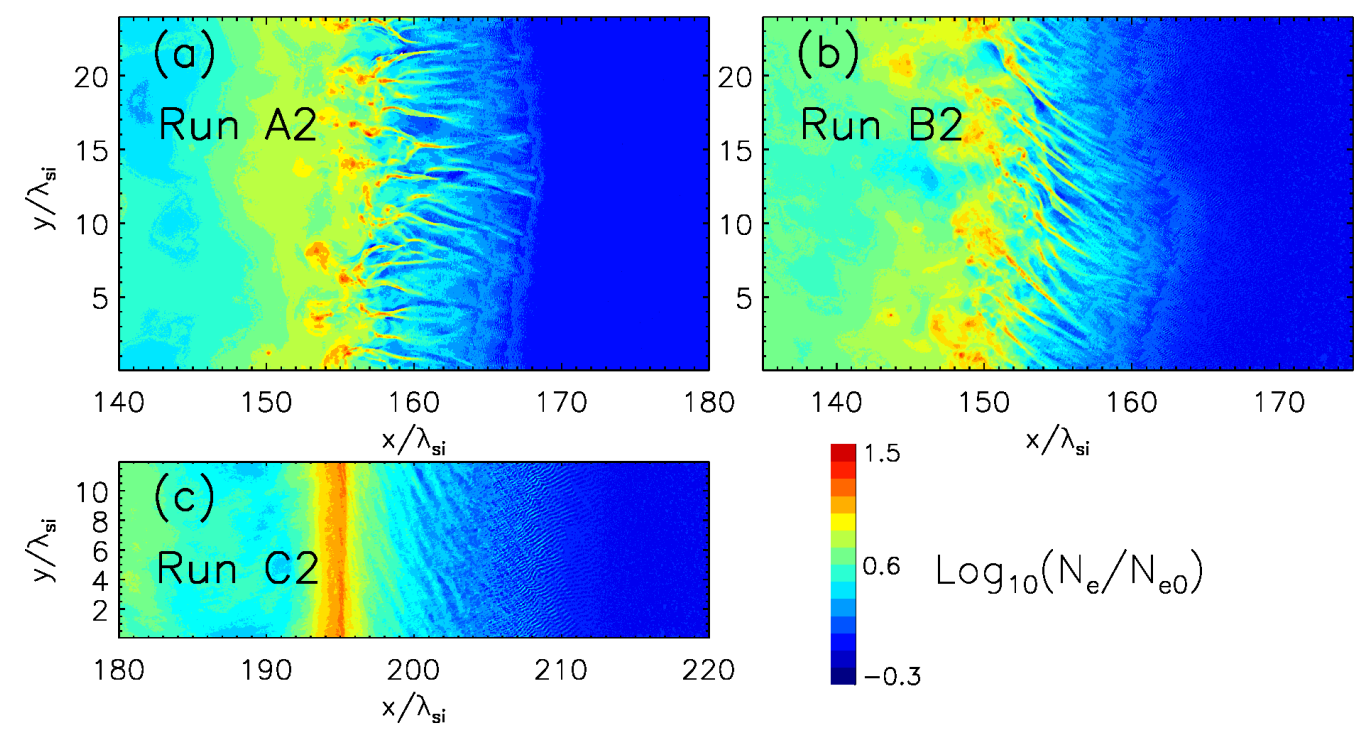

Figure 2: Distributions of the normalized electron number density at shocks propagating in plasmas with $\beta_{p}=0.5$. Panel (a): run $\mathrm{A} 2$ at $t \Omega_{\mathrm{i}}=8.125$, panel (b): run B2 at $t \Omega_{\mathrm{i}}=7.25$, and panel (c): run C2 at $t \Omega_{i}=7.375$. A logarithmic scale is used.

\section{Results}

\subsection{Global Shock Structure}

Figure 2 shows electron density distributions at right shocks, propagating in a moderatetemperature plasma with $\beta_{p}=0.5$ (runs A2, B2, and C2). The time is close to the end of the simulation runs and at a phase of the shock reformation in which the largest number of shockreflected ions appear. These ions determine the shock structure as they interact with the upstream plasma and excite the Buneman instability in the shock foot and the Weibel-type filamentation instability in the shock ramp. One can see that the geometry of the latter depend on the configuration of the uniform magnetic field. This is because the field defines the gyration direction of 
the reflected ions. For both the in-plane and $\varphi=45^{\circ}$ magnetic field configurations (runs A and B) spontaneous magnetic reconnection triggered by merging magnetic filaments is also observed [10]. Ion reflection also leads to the overshoot, i.e., plasma compression at the shock front that exceeds the compression expected MHD jump conditions.
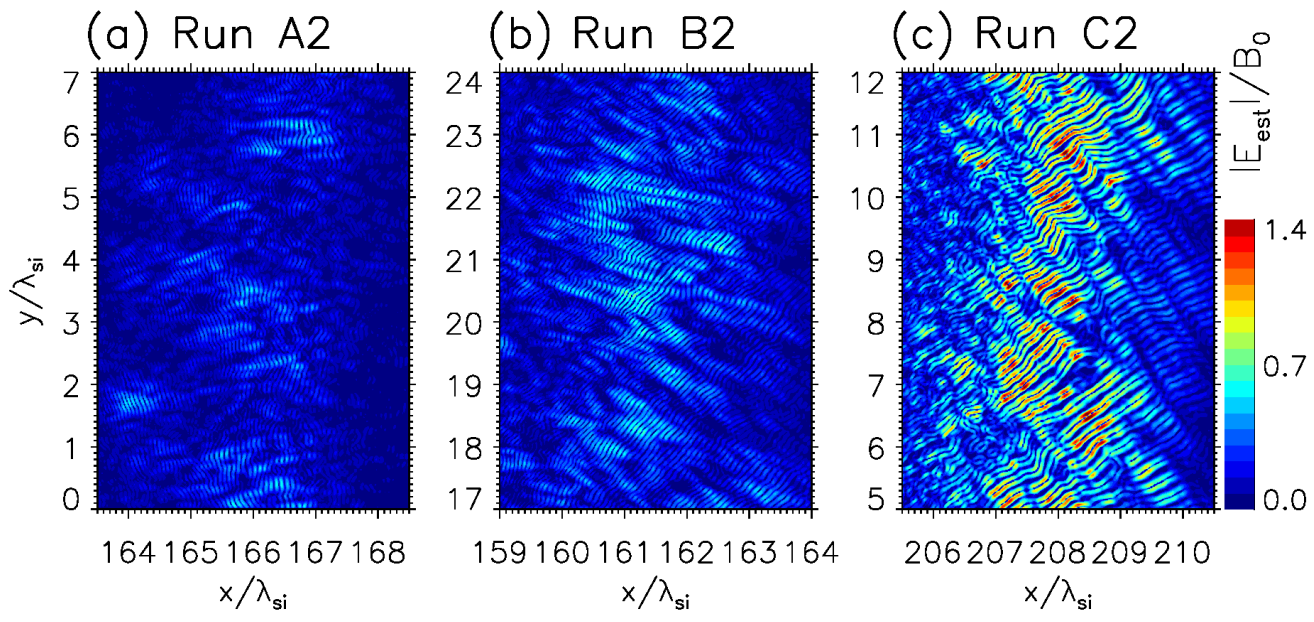

Figure 3: Electrostatic field amplitudes normalized to the upstream magnetic field strength in selected regions of the shock foot with most intense Buneman waves for runs A2 (a), B2 (b), and C2 (c) (see Fig. 2).

Figure 3 zooms-in on a portion of the right shock foot regions shown in Figure 2. The electrostatic field amplitude is calculated as $\left|E_{\text {est }}\right|=|-\nabla \phi|$, where $\phi$ is the electric potential derived directly from the charge distribution. The wave structures occur at electron scales and we identify them with the Buneman waves. The properties of these electrostatic modes depend on physical parameters such as the shock Mach number and plasma temperature. As one can see, in 2D simulations the orientation of the average magnetic field with respect to the simulation plane also plays a role. Recall that the field orientation is the only variable parameter in runs shown in Figure 3. The intensity of the electrostatic waves varies considerably during a shock reformation cycle on account of the changing number of shock-reflected ions. In general though, the region with high-intensity coherent waves is larger and considerably less patchy for the out-of-plane configurations (runs $\mathrm{C}$ ) than it is for an inclined or in-plane magnetic field (runs A and B). Also, in moderate-temperature plasma (runs *2) the peak intensity of Buneman waves is less than that in the cold plasma (runs $* 1$ ), but the surface area occupied by the waves is larger in that case.

\subsection{Electron Acceleration}

The parameters in our simulations should provide suitable conditions for electron acceleration to nonthermal energies. Figure 4 traces trajectories of typical particles energized at the shock for the out-of-plane (left panel, run C2) and in-plane (right panel, run A2) magnetic-field configurations (the case with $\varphi=45^{\circ}$ is similar to that of run A2). In both cases, the acceleration starts with the SSA in the shock foot. Already at this stage the energy acquired by an electron in the Bunemanwave region is larger in run C2 than in run A2 (Figs. 4a1 and 4a2). Subsequent acceleration is also markedly different. In run $\mathrm{C} 2$, the electron increases its energy on the way through the shock ramp, reaching maximum Lorentz factor of $\gamma \simeq 6$ at the overshoot (Figs. $4 \mathrm{~b} 1-\mathrm{c} 1$ ), and losing the 

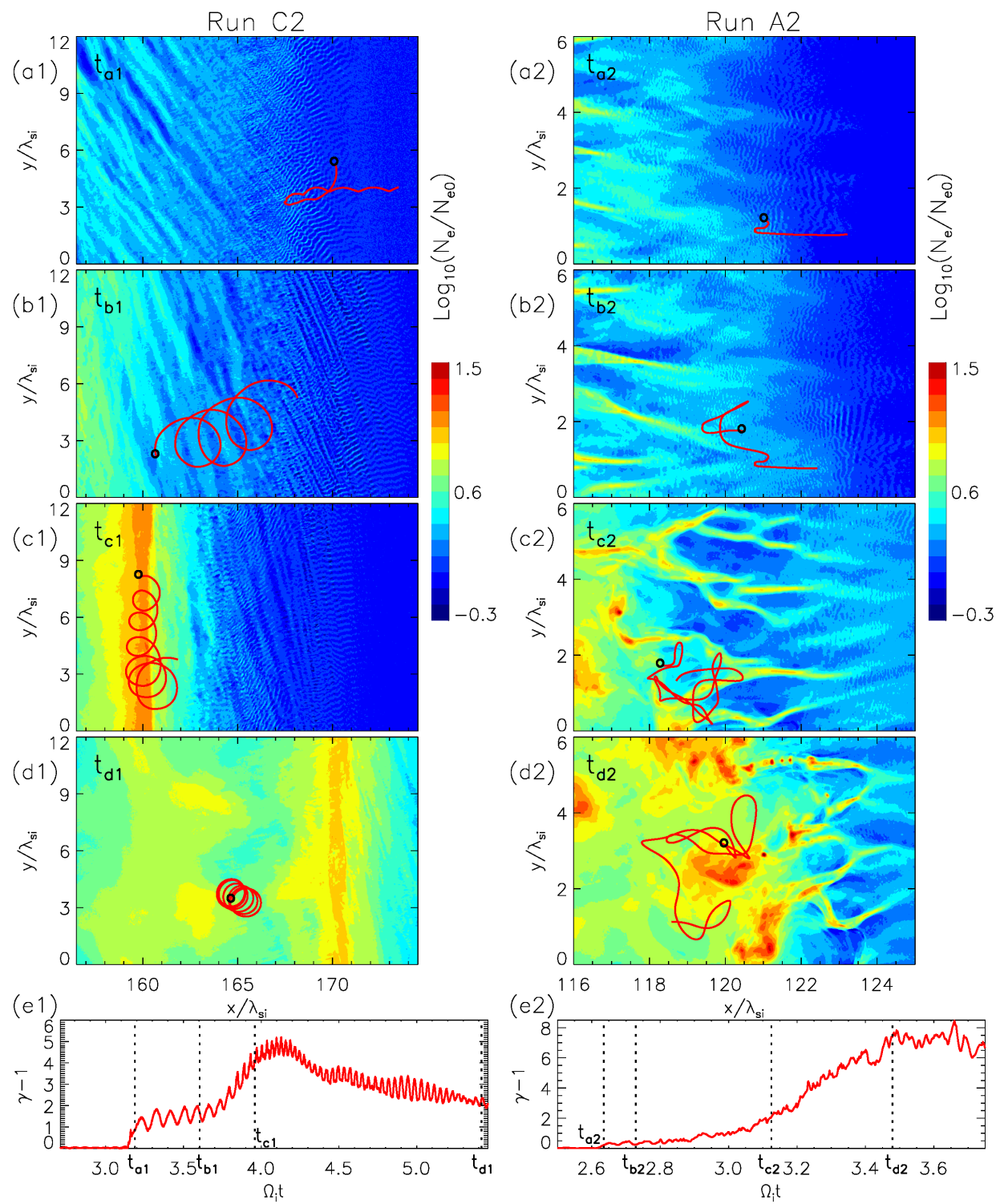

Figure 4: Trajectory segments of sample high-energy electrons from run C2 (left panels *1) and A2 (right panels $* 2$ ) superposed on electron density maps at four time intervals, $t_{a *}-t_{d *}$, in panels $\left(\mathrm{a}^{*}\right)-\left(\mathrm{d}^{*}\right)$, respectively. Black circles indicate the position of electrons at these moments and red lines give their trajectory histories for the time span $0.2 \Omega_{i}^{-1}$. Time intervals are also marked in panels (e*), showing the temporal development of the kinetic energy of the electrons. Normalized electron density uses logarithmic scaling.

energy in the downstream. The energy variations are thus commensurate with plasma compression around the shock and the acceleration beyond the SSA phase is therefore largely adiabatic. On the other hand, in run A2 the particle in the shock ramp interacts many times with moving magnetic structures (Figs. 4b2-c2) and undergoes a stochastic (second-order Fermi) acceleration processes. It reaches a maximum energy of about $\gamma \simeq 9$ in the shock overshoot (Fig. $4 \mathrm{~d} 2$ ) and retains that energy in the downstream. Electron injection in this case thus mainly involves irreversible non-adiabatic processes. 


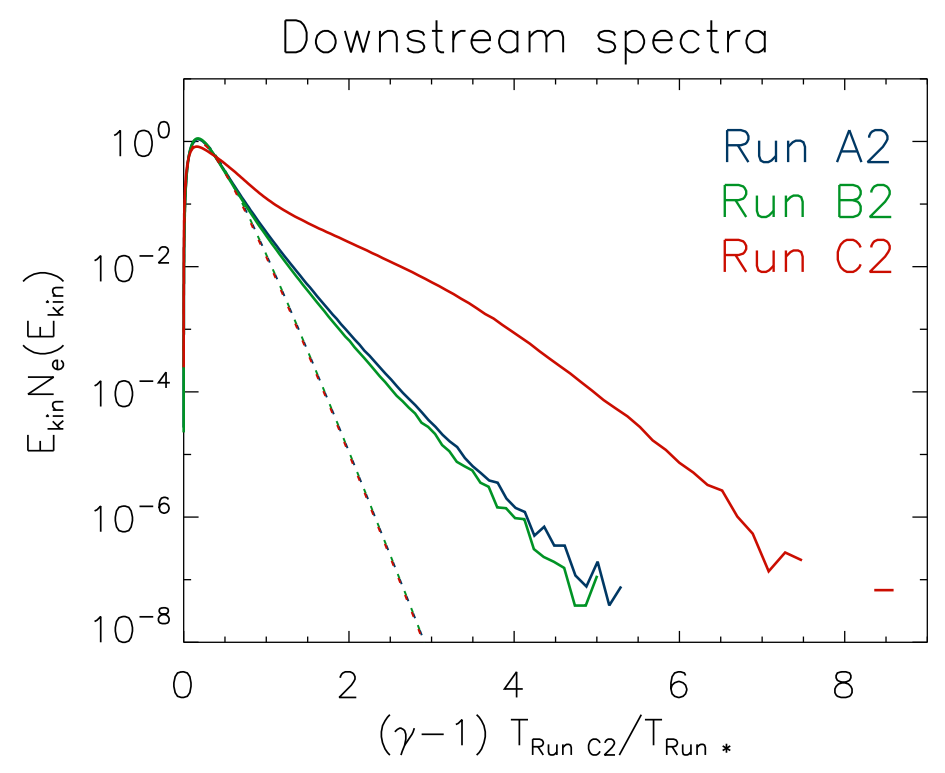

Figure 5: Rescaled downstream spectra for runs A2 (blue), B2 (green) and C2 (red). The dashed lines represent fits of a relativistic Maxwellian to the low-energy spectra.

\subsection{Spectra in the downstream region}

Figure 5 shows downstream electron spectra for runs A2, B2 and C2, rescaled in units of the downstream temperature. Nonthermal electron fractions (NEFs) are provided in Table 1. Shock self-reformation leads to temporal variations in the shock structure. In consequence, the electron energization becomes time-dependent and NEF shows quasi-periodic fluctuations in the downstream region. Electron spectra in Figure 5 contain particles processed over two cycles of the shock reformation. One can note, that runs with the out-of-plane magnetic-field configuration produce a much higher NEFs than are found for $\varphi=45^{\circ}$ or $\varphi=0^{\circ}$. For a given magnetic-field orientation, we also observe a higher nonthermal fraction at shocks propagating into moderate- $\beta_{\mathrm{p}}$ plasma than for low $\beta_{\mathrm{p}}$.

\section{Discussion}

Our 2D3V PIC simulations of electron injection at non-relativistic perpendicular collisionless shocks with high Alfvénic Mach number differ in plasma temperature and the configuration the large-scale magnetic field with respect to the simulation plane, but all assume parameters that should permit efficient electron acceleration. The field orientation imposes differences in the Buneman wave and magnetic filament structure. One effect that results is that magnetic reconnection is observed in the shock ramp only for simulations with $\varphi=0^{\circ}$ and $\varphi=45^{\circ}$ magnetic-field configurations. On the other hand, the cases with $\varphi=90^{\circ}$ provide higher intensity of the Buneman waves at the shock foot than the other two configurations, and the waves are coherent and found in a larger region. In consequence, the number of particles energized in the Buneman wave zone is orders of magnitude larger for the out-of-plane configurations than in cases with $\varphi=0^{\circ}$ and $\varphi=45^{\circ}$. The SSA is followed by further acceleration in the shock ramp and the overshoot, whose 
nature depends on the angle $\varphi$. However, as in all simulations the Buneman instability serves as injector, the fraction of nonthermal electrons is more then 10 times larger for $\varphi=90^{\circ}$ than for the other configurations on account of the higher SSA efficiency in the foot region. Similarly, due to larger area with Buneman waves formed in moderate- $\beta_{\mathrm{p}}$ plasmas, electrons experience more SSA and resulting NEF is larger in this case than for the cold plasmas.

We have presented results of $2 \mathrm{D} 3 \mathrm{~V}$ simulations, but the real world is $3 \mathrm{D}$ throughout and the question arises which of the $2 \mathrm{D} 3 \mathrm{~V}$ configurations we have studied provides the closest match to the 3D case. For the out-of-plane magnetic field orientation, particles are mostly confined to the simulation plane and their 3D motion is not fully accounted for. Therefore, the low fraction of nonthermal electrons produced in $\varphi=0^{\circ}$ and $\varphi=45^{\circ}$ simulations seems to be a better indicator of the 3D injection efficiency than is the high abundance of energetic electrons for $\varphi=90^{\circ}$. True 3D simulations are urgently needed to resolve this issue.

\section{Acknowledgments}

The work of A.B., J.N. and O.K. is supported by Narodowe Centrum Nauki through research project DEC-2013/10/E/ST9/00662. M.P. acknowledges support through grants PO 1508/1-1 and PO 1508/1-2 of the Deutsche Forschungsgemeinschaft. Numerical simulations have been performed on the Prometheus system at ACC Cyfronet AGH. Part of the numerical work was also conducted on resources provided by The North-German Supercomputing Alliance (HLRN) under project bbp00003.

\section{References}

[1] Wieland, V., Pohl, M., Niemiec, J., Rafighi, I., \& Nishikawa, K.-I. 2016, ApJ, 820, 62

[2] Umeda, T., Yamao, M., \& Yamazaki, R. 2008, ApJ Lett., 681, L85

[3] Burgess, D., \& Scholer, M. 2007, Physics of Plasmas, 14, 012108

[4] Buneman, O. 1958, Phys. Rev. Lett,1,8

[5] Hoshino, M., \& Shimada, N. 2002, ApJ, 572, 880

[6] Amano, T., \& Hoshino, M. 2009, ApJ, 690, 244

[7] Matsumoto, Y., Amano, T., \& Hoshino, M. 2012, ApJ, 755, 109

[8] Matsumoto, Y., Amano, T., \& Hoshino, M. 2013, Physical Review Letters, 111, 215003

[9] Bohdan, A., Niemiec, J., Kobzar, O., Pohl, M. 2017, ApJ (submitted)

[10] Matsumoto, Y., Amano, T., Kato, T. N., \& Hoshino, M. 2015, Science, 347, 974

[11] Buneman, O. 1993, in Computer Space Plasma Physics: Simulation Techniques and Software, Eds.: Matsumoto \& Omura, Tokyo: Terra, p.67

[12] Niemiec, J., Pohl, M., Stroman, T., \& Nishikawa, K.-I. 2008, ApJ, 684, 1174-1189 